\title{
Asymptotics for the Solutions to Defective Renewal Equations
}

\author{
Kaiyong Wang, ${ }^{1,2}$ Yang Chen, ${ }^{1}$ and Zhongquan Tan $^{3}$ \\ ${ }^{1}$ School of Mathematics and Physics, Suzhou University of Science and Technology, Suzhou 215009, China \\ ${ }^{2}$ Department of Mathematics, Southeast University, Nanjing 210096, China \\ ${ }^{3}$ College of Mathematics, Physics and Information Engineering, Jiaxing University, Jiaxing 314001, China
}

Correspondence should be addressed to Kaiyong Wang; beewky@163.com

Received 14 November 2013; Accepted 12 March 2014; Published 6 April 2014

Academic Editor: Yiming Ding

Copyright (C) 2014 Kaiyong Wang et al. This is an open access article distributed under the Creative Commons Attribution License, which permits unrestricted use, distribution, and reproduction in any medium, provided the original work is properly cited.

This paper investigates the defective renewal equations under the nonconvolution equivalent distribution class. The asymptotics of the solution to the defective renewal equations have been given for the heavy-tailed and light-tailed cases, respectively.

\section{Introduction}

This paper will consider the defective renewal equation

$$
Z(x)=z(x)+q \int_{0}^{x} Z(x-y) F(\mathrm{~d} y), \quad x \geq 0,
$$

where $F$ is a proper distribution on $[0, \infty), z(x) \geq 0$ is a known and locally bounded function on $[0, \infty)$, and $0<q<$ 1 . The only solution $Z(x)$ to (1) is given by

$$
Z(x)=(1-q)^{-1} \int_{0}^{x} z(x-y) U_{0}(\mathrm{~d} y), \quad x \geq 0,
$$

where

$$
U_{0}(x)=(1-q) \sum_{n=0}^{\infty} q^{n} F^{* n}(x), \quad x \geq 0
$$

(see e.g., Asmussen [1, Chapter V]) and here $F^{* n}$ is the $n$-fold convolution of $F$ with itself, $n \geq 2, F^{* 1}=F$, and $F^{* 0}$ is the distribution degenerate at zero.

Since in most cases it is not easy to calculate (2), more attention is paid to the asymptotics of the solution $Z(x)$. When $Z=F$, the asymptotics of $Z(x)$ have been investigated by many researchers, such as Embrechts et al. [2], Embrechts and Goldie [3], and Cline [4]. Asmussen [5] and Asmussen et al. [6] considered the case that $z$ is a subexponential density. Yin and Zhao [7] obtained the asymptotics of $Z(x)$ for the monotone function $z$. For the above case, K. Wang and Y.
Wang [8] gave the local asymptotics of $Z(x)$. Cui et al. [9] considered a new case that

$$
\lim _{x \rightarrow \infty} \frac{z(x)}{\bar{F}(x)}=c
$$

where $c$ is a positive constant. In Corollary 5.1 and Theorem 5.2 of Cui et al. [9], they obtained the asymptotics of $Z(x)$ under the condition that $F \in \mathcal{S}$ and $F \in \mathcal{S}(\gamma)$ for some $\gamma>0$ with $\widehat{F}(\gamma)=\int_{-\infty}^{\infty} e^{\gamma u} F(\mathrm{~d} u)<1$, respectively. The classes $\mathcal{S}$ and $(\gamma), \gamma>0$, (the definitions of these distribution classes will be given below) are convolution equivalent distribution classes. But beyond the convolution equivalent distribution classes, there exist some other distributions. How to estimate the asymptotics of the solution $Z(x)$ for the nonconvolution equivalent distribution $F$ will be an interesting question. This paper will investigate this case. Under the conditions (4) and that $F$ may not belong to the convolution equivalent distribution class, this paper obtains the asymptotics of the solution $Z(x)$. In order to better illuminate our motivation and results, we will introduce some notions and notation.

Without special statement, in this paper a limit is taken as $x \rightarrow \infty$. For two nonnegative functions $a(\cdot)$ and $b(\cdot)$, we write $a(x) \lesssim b(x)$ if $\lim \sup a(x) / b(x) \leq 1$, write $a(x) \geq b(x)$ if $\lim \inf a(x) / b(x) \geq 1$, write $a(x) \sim b(x)$ if $\lim a(x) / b(x)=$ 1 , and write $a(x)=o(b(x))$ if $\lim a(x) / b(x)=0$. For a proper distribution $V$ on $(-\infty, \infty)$, the tail of $V$ is $\bar{V}=1-V$. For a real number $\gamma$, denote by $\widehat{V}(\gamma)=\int_{-\infty}^{\infty} e^{\gamma u} V(\mathrm{~d} u)$ the moment generating function of $V$. 
Firstly, we will introduce some heavy-tailed and lighttailed distribution classes. Say that a random variable (r.v.) $\xi$ (or its corresponding distribution $V$ ) is heavy-tailed if for all $\lambda>0, \widehat{V}(\gamma)=\infty$; otherwise, say that it is light-tailed. Let $V$ be a distribution on $(-\infty, \infty)$. Say that the distribution $V$ belongs to the class $\mathscr{L}(\gamma)$ for some $\gamma \geq 0$, if for any $t \in(-\infty, \infty)$,

$$
\bar{V}(x-t) \sim e^{\gamma t} \bar{V}(x),
$$

where, when $\gamma>0$ and $V$ is a lattice distribution, $x$ and $t$ are both taken as a multiple of the lattice step. Say that the distribution $V$ belongs to the class $\mathcal{S}(\gamma)$ for some $\gamma \geq 0$, if $V \in \mathscr{L}(\gamma), \widehat{V}(\gamma)<\infty$, and

$$
\overline{V^{* 2}}(x) \sim 2 \widehat{V}(\gamma) \bar{V}(x) .
$$

The class $\mathcal{S}(\gamma), \gamma \geq 0$, is called the convolution equivalent distribution class and was introduced by [10] and Chover et al. $[11,12]$ for distributions on $[0, \infty)$ and by Pakes [13] for distributions on $(-\infty, \infty)$. Especially, we call $\mathcal{S}(0)$ and $\mathscr{L}(0)$ the subexponential distribution class and the longtailed distribution class, denoted by $\mathcal{S}$ and $\mathscr{L}$, respectively.

This paper will mainly investigate the case that the distribution $F$ may not be convolution equivalent. We will introduce another distribution class. Say that the distribution $V$ belongs to the class $\mathcal{O} \mathcal{S}$, if $\bar{V}(x)>0$ for sufficiently large $x$ and

$$
C_{V}^{*}=\limsup _{x \rightarrow \infty} \frac{\overline{V^{2 *}}(x)}{\bar{V}(x)}<\infty .
$$

Clearly, if $V \in \mathcal{S}(\gamma)$ for some $\gamma \geq 0$ then $C_{V}^{*}=2 \widehat{V}(\gamma)$. Therefore, for each $\gamma \geq 0, \mathcal{S}(\gamma) \subset \mathcal{O} \mathcal{S}$. If $V \in \mathscr{L}(\gamma)$ for some $\gamma \geq 0$ then $C_{V}^{*} \geq 2 \widehat{V}(\gamma)$, which can be obtained by Lemma 2.4 of Embrechts and Goldie [3] and Theorems 1.1 and 1.2 of $\mathrm{Yu}$ et al. [14]. The class $\mathcal{O} \mathcal{S}$ is first introduced by Klüppelberg [15] and detailedly studied in Klüppelberg and Villasenor [16], Shimura and Watanabe [17], Watanabe and Yamamura [18], Lin and Wang [19], Yang and Wang [20], and Wang et al. [21], among others. This paper will consider the case that $F \in \mathscr{L}(\gamma) \cap \mathcal{O} \mathcal{S}, \gamma \geq 0$. As noted by Wang et al. [21], for each $\gamma>0, \mathcal{S}(\gamma) \subset \mathscr{L}(\gamma) \cap \mathcal{O} \mathcal{S}$ and the class $(\mathscr{L}(\gamma) \cap \mathcal{O} \mathcal{S}) \backslash \mathcal{S}(\gamma)$ is nonempty.

We first present the main result for the heavy-tailed case.

Theorem 1. For the renewal equation (1), assume that (4) holds. If $F \in \mathscr{L} \cap \mathcal{O} \mathcal{S}$ and $C_{F}^{*}<1+q^{-1}$, then

$$
\frac{c}{1-q} \bar{F}(x) \lesssim Z(x) \lesssim\left(\frac{1}{1-q}+\frac{C_{U_{0}}^{*}-2}{1-q\left(C_{F}^{*}-1\right)}\right) c \bar{F}(x) \text {. }
$$

Remark 2. If $F \in \mathcal{S}$ then $U_{0} \in \mathcal{S}$ by Theorem 1 of Cline [4]. Hence, $C_{U_{0}}^{*}=2$. The result of Theorem 1 implies that

$$
Z(x) \sim \frac{c}{1-q} \bar{F}(x),
$$

which is Corollary 5.1 (ii) of Cui et al. [9].
In the following, we give the result for the light-tailed case.

Theorem 3. For the renewal equation (1), assume that (4) holds. If $F \in \mathscr{L}(\gamma) \cap \mathcal{O} \mathcal{S}$ for some $\gamma>0$ satisfying $\widehat{F}(\gamma)<1$ and $C_{F}^{*}<1+\widehat{F}(\gamma)$, then

$$
c_{1} \bar{F}(x) \lesssim Z(x) \lesssim c_{2} \bar{F}(x),
$$

where

$$
\begin{gathered}
c_{1}=\frac{c}{1-q \widehat{F}(\gamma)}+\frac{q I}{(1-q \widehat{F}(\gamma))^{2}}, \\
c_{2}=\frac{c}{1-q \widehat{F}(\gamma)}+\frac{c(1-q \widehat{F}(\gamma))}{1-q\left(C_{F}^{*}-\widehat{F}(\gamma)\right)}\left(\frac{C_{U_{0}}^{*}}{1-q}-\frac{2}{1-q \widehat{F}(\gamma)}\right) \\
+\frac{q I}{\left(1-q\left(C_{F}^{*}-\widehat{F}(\gamma)\right)\right)(1-q \widehat{F}(\gamma))}, \\
I=\gamma \int_{0}^{\infty} e^{\gamma y} z(y) \mathrm{d} y .
\end{gathered}
$$

Remark 4. If $F \in \mathcal{S}(\gamma)$ for some $\gamma>0$, by Theorem 1 of Cline [4], then $C_{F}^{*}=2 \widehat{F}(\gamma)$ and $U_{0} \in \mathcal{S}(\gamma)$. Therefore, $C_{U_{0}}^{*}=2 \widehat{U_{0}}(\gamma)=2(1-q) /(1-q \widehat{F}(\gamma))$. Then we can obtain from Theorem 3 that

$$
Z(x) \sim c_{1} \bar{F}(x),
$$

which is Theorem 5.2 (ii) of Cui et al. [9].

\section{Proofs of Theorems}

Before giving the proof of Theorems 1 and 3, we first give some lemmas. The first lemma comes from Lemma 2.2 of Yu and Wang [22], which will need the following notation. For a distribution $V$ on $(-\infty, \infty)$ and any $\gamma \geq 0$, define

$$
\begin{gathered}
\mathscr{H}_{V}(\gamma)=\left\{h \text { on }[0, \infty): h(x) \uparrow \infty, x^{-1} h(x) \longrightarrow 0,\right. \\
\bar{V}(x-y) \sim e^{\gamma y} \bar{V}(x) \\
\text { uniformly for }|y| \leq h(x)\} .
\end{gathered}
$$

Lemma 5. Suppose that $V$ is a distribution on $(-\infty, \infty)$ and belongs to the class $\mathscr{L}(\gamma) \cap \mathcal{O} \mathcal{S}$ for some $\gamma \geq 0$. Then for any $h \in \mathscr{H}_{V}(\gamma)$,

$$
\limsup \int_{h(x)}^{x-h(x)} \frac{\bar{V}(x-u)}{\bar{V}(x)} V(d u)=C_{V}^{*}-2 \widehat{V}(\gamma) .
$$

Lemma 6. For the random sum (3), assume that $F \in \mathscr{L}(\gamma) \cap$ $\sigma \mathcal{S}$ for some $\gamma \geq 0$. When $\gamma=0$, let $C_{F}^{*}<1+q^{-1}$; when $\gamma>0$, let $\widehat{F}(\gamma)<1$ and $C_{F}^{*}<1+\widehat{F}(\gamma)$. Then $U_{0} \in \mathscr{L}(\gamma) \cap \mathcal{O} \mathcal{S}$,

$$
\begin{gathered}
\liminf \frac{\overline{U_{0}}(x)}{\bar{F}(x)}=\frac{q(1-q)}{(1-q \widehat{F}(\gamma))^{2}}, \\
\limsup \frac{\overline{U_{0}}(x)}{\bar{F}(x)} \leq \frac{q(1-q)}{\left(1-q\left(C_{F}^{*}-\widehat{F}(\gamma)\right)\right)(1-q \widehat{F}(\gamma))} .
\end{gathered}
$$


Proof. We first prove (15). Let $\tau$ be a r.v. with a distribution $P(\tau=n)=(1-q) q^{n}, n=0,1, \ldots$. When $\gamma=0$ and since $F$ is heavy-tailed and $\tau$ is light-tailed, by Theorem 2 of Denisov et al. [23], it holds that

$$
\lim \inf \frac{\overline{U_{0}}(x)}{\bar{F}(x)}=\frac{q}{(1-q)} .
$$

When $\gamma>0$ and since there exists $\epsilon_{1}>0$ such that

$$
E\left(\max \left\{\widehat{F}(\gamma)+\epsilon_{1}, 1\right\}\right)^{\tau}<\infty,
$$

by Theorem 1.2 of Yu et al. [14], it holds that

$$
\begin{aligned}
\liminf \frac{\overline{U_{0}}(x)}{\bar{F}(x)} & \leq E \tau(\widehat{F}(\gamma))^{\tau-1} \\
& =\frac{q(1-q)}{(1-q \widehat{F}(\gamma))^{2}} .
\end{aligned}
$$

On the other hand, since $F \in \mathscr{L}(\gamma)$, by Fatou's lemma and Lemma 5.4 of Pakes [13], we have

$$
\begin{aligned}
\liminf \frac{\overline{U_{0}}(x)}{\bar{F}(x)} & \geq E \tau(\widehat{F}(\gamma))^{\tau-1} \\
& =\frac{q(1-q)}{(1-q \widehat{F}(\gamma))^{2}} .
\end{aligned}
$$

This completes the proof of (15).

Now we prove (16). Since $C_{F}^{*}<1+q^{-1}$ for $\gamma=0$ and $C_{F}^{*}<1+\widehat{F}(\gamma)$ for $\gamma>0$, there exists $\epsilon_{2}>0$ such that for $\gamma \geq 0$

$$
E\left(C_{F}^{*}-\widehat{F}(\gamma)+\epsilon_{2}\right)^{\tau}<\infty
$$

Hence, by Corollary 1 of Yu and Wang [22], we get $U_{0} \in$ $\mathscr{L}(\gamma) \cap \mathscr{O} \mathcal{S}$ and (16) holds.

Proof of Theorem 1. Since $F \in \mathscr{L} \cap \mathcal{O} \mathcal{S}$, we get $U_{0} \in \mathscr{L} \cap \mathcal{O} \mathcal{S}$ by Lemma 6 . For any fixed positive constant $M$, when $x$ is sufficiently large, we get

$$
\begin{aligned}
Z(x) & =(1-q)^{-1}\left(\int_{0}^{M}+\int_{M}^{x-M}+\int_{x-M}^{x}\right) z(x-y) U_{0}(\mathrm{~d} y) \\
& =: J_{1}(x, M)+J_{2}(x, M)+J_{3}(x, M) .
\end{aligned}
$$

By (4) and $F \in \mathscr{L}$, we get

$$
\begin{aligned}
J_{1}(x, M) & \sim c(1-q)^{-1} \int_{0}^{M} \bar{F}(x-y) U_{0}(\mathrm{~d} y) \\
& \sim c(1-q)^{-1} \bar{F}(x) U_{0}(M) .
\end{aligned}
$$

Hence,

$$
\lim _{M \rightarrow \infty} \lim _{x \rightarrow \infty} \frac{J_{1}(x, M)}{\bar{F}(x)}=\frac{c}{1-q} .
$$

For $J_{3}(x, M)$, since $\sup _{t \in[0, M]} z(t)<\infty$, by $U_{0} \in \mathscr{L}$ and Lemma 6, it holds that

$$
\begin{aligned}
J_{3}(x, M) & \leq(1-q)^{-1} \sup _{t \in[0, M]} z(t)\left(\overline{U_{0}}(x-M)-\overline{U_{0}}(x)\right) \\
& =o\left(\overline{U_{0}}(x)\right)=o(\bar{F}(x)) .
\end{aligned}
$$

For $J_{2}(x, M)$, we first estimate the asymptotics of

$$
\int_{M}^{x-M} \overline{U_{0}}(x-y) U_{0}(\mathrm{~d} y)
$$

as firstly letting $x \rightarrow \infty$ and then letting $M \rightarrow \infty$. For any $x \geq 0$, it holds that

$$
\begin{aligned}
\overline{U^{* 2}}(x)= & 2 \int_{0}^{M} \overline{U_{0}}(x-y) U_{0}(\mathrm{~d} y) \\
& +\int_{M}^{x-M} \overline{U_{0}}(x-y) U_{0}(\mathrm{~d} y) \\
& +\overline{U_{0}}(x-M) \overline{U_{0}}(M) .
\end{aligned}
$$

Since $U_{0} \in \mathscr{L}$, we have

$$
\begin{gathered}
\lim _{M \rightarrow \infty} \lim _{x \rightarrow \infty} \int_{0}^{M} \frac{\overline{U_{0}}(x-y)}{\overline{U_{0}}(x)} U_{0}(\mathrm{~d} y)=1, \\
\lim _{M \rightarrow \infty} \lim _{x \rightarrow \infty} \frac{\overline{U_{0}}(x-M) \overline{U_{0}}(M)}{\overline{U_{0}}(x)}=0 .
\end{gathered}
$$

Hence, $U_{0} \in \mathcal{O} \mathcal{S}$ means that

$$
\limsup _{M \rightarrow \infty} \limsup _{x \rightarrow \infty} \int_{M}^{x-M} \frac{\overline{U_{0}}(x-y)}{\overline{U_{0}}(x)} U_{0}(\mathrm{~d} y)=C_{U_{0}}^{*}-2,
$$

which, combining with (4) and Lemma 6, yields that

$$
\begin{aligned}
\limsup _{M \rightarrow \infty} \limsup _{x \rightarrow \infty} \frac{J_{2}(x, M)}{\bar{F}(x)} \\
\leq \frac{c}{1-q\left(C_{F}^{*}-1\right)} \\
\quad \times \limsup _{M \rightarrow \infty} \limsup _{x \rightarrow \infty} \int_{M}^{x-M} \frac{\overline{U_{0}}(x-y)}{\overline{U_{0}}(x)} U_{0}(\mathrm{~d} y) \\
=\frac{c}{1-q\left(C_{F}^{*}-1\right)}\left(C_{U_{0}}^{*}-2\right) .
\end{aligned}
$$

Hence, (8) can be obtained by (22)-(25) and (30).

Proof of Theorem 3. It follows from Lemma 6 and $F \in \mathscr{L}(\gamma) \cap$ $\mathcal{O} \mathcal{S}$ that $U_{0} \in \mathscr{L}(\gamma) \cap \mathcal{O} \mathcal{S}$. Taking $h \in \mathscr{H}_{U_{0}}(\gamma)$, when $x$ is sufficiently large, we get

$$
\begin{aligned}
Z(x)= & (1-q)^{-1}\left(\int_{0}^{h(x)}+\int_{h(x)}^{x-h(x)}+\int_{x-h(x)}^{x}\right) \\
& \times z(x-y) U_{0}(\mathrm{~d} y) \\
= & : I_{1}(x)+I_{2}(x)+I_{3}(x) .
\end{aligned}
$$


By (4) and $F \in \mathscr{L}(\gamma)$, we have

$$
\begin{aligned}
I_{1}(x) & \sim c(1-q)^{-1} \int_{0}^{h(x)} \bar{F}(x-y) U_{0}(\mathrm{~d} y) \\
& \sim \frac{c}{1-q \widehat{F}(\gamma)} \bar{F}(x) .
\end{aligned}
$$

By (4) and Lemmas 5 and 6, it holds that

$$
\begin{aligned}
I_{2}(x) & \sim c(1-q)^{-1} \int_{h(x)}^{x-h(x)} \bar{F}(x-y) U_{0}(\mathrm{~d} y) \\
& \leqslant \frac{c(1-q \widehat{F}(\gamma))^{2}}{q(1-q)^{2}} \int_{h(x)}^{x-h(x)} \overline{U_{0}}(x-y) U_{0}(\mathrm{~d} y) \\
& \leqslant \frac{c(1-q \widehat{F}(\gamma))^{2}}{q(1-q)^{2}}\left(C_{U_{0}}^{*}-2 \widehat{U_{0}}(\gamma)\right) \overline{U_{0}}(x) \\
& \leqslant \frac{c(1-q \widehat{F}(\gamma))}{1-q\left(C_{F}^{*}-\widehat{F}(\gamma)\right)}\left(\frac{C_{U_{0}}^{*}}{1-q}-\frac{2}{1-q \widehat{F}(\gamma)}\right) \bar{F}(x) .
\end{aligned}
$$

For $I_{3}(x)$, using Lemma 6 and the way of dealing with $Z_{3}(x)$ in Theorem 5.2 of Cui et al. [9], we can get

$$
\begin{aligned}
& \limsup \frac{I_{3}(x)}{\bar{F}(x)} \leq \frac{q I}{\left(1-q\left(C_{F}^{*}-\widehat{F}(\gamma)\right)\right)(1-q \widehat{F}(\gamma))}, \\
& \liminf \frac{I_{3}(x)}{\bar{F}(x)} \geq \frac{q I}{(1-q \widehat{F}(\gamma))^{2}} .
\end{aligned}
$$

Hence, (10) can be obtained by (31)-(34).

\section{Conflict of Interests}

The authors declare that there is no conflict of interests regarding the publication of this paper.

\section{Acknowledgments}

The authors wish to thank the editor and the referees for their very valuable comments by which the presentation of the paper has been improved. This work is supported by the National Natural Science Foundation of China, Tian Yuan Foundation (no. 11226211), the Natural Science Foundation of Jiangsu Province (no. BK2012165), China Postdoctoral Science Foundation (no. 2012M520963), and the Research Foundation of SUST.

\section{References}

[1] S. Asmussen, Applied Probability and Queues, vol. 51 of Applications of Mathematics, Springer, New York, NY, USA, 2nd edition, 2003.

[2] P. Embrechts, C. M. Goldie, and N. Veraverbeke, "Subexponentiality and infinite divisibility," Zeitschrift für Wahrscheinlichkeitstheorie und Verwandte Gebiete, vol. 49, no. 3, pp. 335347, 1979.
[3] P. Embrechts and C. M. Goldie, "On convolution tails," Stochastic Processes and Their Applications, vol. 13, no. 3, pp. 263-278, 1982.

[4] D. B. H. Cline, "Convolutions of distributions with exponential and subexponential tails," Australian Mathematical Society Journal A: Pure Mathematics and Statistics, vol. 43, no. 3, pp. 347-365, 1987.

[5] S. Asmussen, "A probabilistic look at the Wiener-Hopf equation," SIAM Review, vol. 40, no. 2, pp. 189-201, 1998.

[6] S. Asmussen, S. Foss, and D. Korshunov, "Asymptotics for sums of random variables with local subexponential behaviour," Journal of Theoretical Probability, vol. 16, no. 2, pp. 489-518, 2003.

[7] C. Yin and J. Zhao, "Nonexponential asymptotics for the solutions of renewal equations, with applications," Journal of Applied Probability, vol. 43, no. 3, pp. 815-824, 2006.

[8] K. Wang and Y. Wang, "Equivalent conditions of local asymptotics for the solutions of defective renewal equations, with applications," Acta Mathematicae Applicatae Sinica (English Series), vol. 26, no. 3, pp. 503-512, 2010.

[9] Z. Cui, Y. Wang, and K. Wang, "Asymptotics for the moments of the overshoot and undershoot of a random walk," Advances in Applied Probability, vol. 41, no. 2, pp. 469-494, 2009.

[10] V. P. Chistyakov, "A theorem on sums of independent positive random variablesand its applications to branching process," Theory of Probability and Its Applications, vol. 9, no. 4, pp. 640648, 1964.

[11] J. Chover, P. Ney, and S. Wainger, "Degeneracy properties of subcritical branching processes," Annals of Probability, vol. 1, pp. 663-673, 1973

[12] J. Chover, P. Ney, and S. Wainger, "Functions of probability measures," Journal d'Analyse Mathématique, vol. 26, pp. 255302, 1973.

[13] A. G. Pakes, "Convolution equivalence and infinite divisibility," Journal of Applied Probability, vol. 41, no. 2, pp. 407-424, 2004.

[14] C. Yu, Y. Wang, and Z. Cui, "Lower limits and upper limits for tails of random sums supported on $\mathbb{R}$," Statistics and Probability Letters, vol. 80, no. 13-14, pp. 1111-1120, 2010.

[15] C. Klüppelberg, "Asymptotic ruin probabilities and hazard rates," Mathematics of Operations Research, vol. 60, pp. 567-576, 1990.

[16] C. Klüppelberg and J. A. Villasenor, "The full solution of the convolution closure problem for convolution-equivalent distributions," Journal of Mathematical Analysis and Applications, vol. 160, no. 1, pp. 79-92, 1991.

[17] T. Shimura and T. Watanabe, "Infinite divisibility and generalized subexponentiality," Bernouli, vol. 11, no. 3, pp. 445-469, 2005.

[18] T. Watanabe and K. Yamamura, "Ratio of the tail of an infinitely divisible distribution on the line to that of its Lévy measure," Electronic Journal of Probability, vol. 15, no. 2, pp. 44-74, 2010.

[19] J. Lin and Y. Wang, "New examples of heavy-tailed Osubexponential distributions and related closure properties," Statistics and Probability Letters, vol. 82, no. 3, pp. 427-432, 2012.

[20] Y. Yang and K. Wang, "Estimates for the tail probability of the supremum of a random walk with independent increments," Chinese Annals of Mathematics B, vol. 32, no. 6, pp. 847-856, 2011.

[21] K. Wang, Y. Yang, and C. Yu, "Estimates for the overshoot of a random walk with negative drift and non-convolution equivalent increments," Statistics and Probability Letters, vol. 83, no. 6, pp. 1504-1512, 2013. 
[22] C. Yu and Y. Wang, "Tail behavior of supremum of a random walk when Cramér's condition fails," Frontiers of Mathematics in China, vol. 9, no. 2, pp. 431-453, 2014.

[23] D. Denisov, S. Foss, and D. Korshunov, "On lower limits and equivalences for distribution tails of randomly stopped sums," Bernoulli, vol. 14, no. 2, pp. 391-404, 2008. 


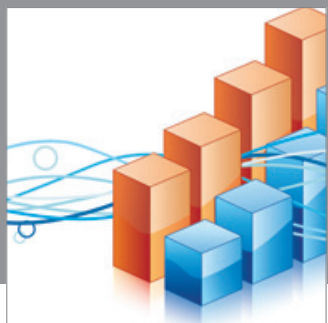

Advances in

Operations Research

mansans

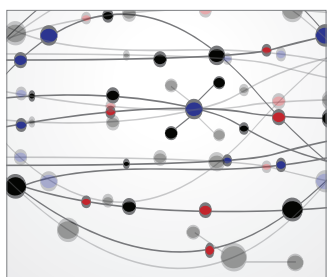

The Scientific World Journal
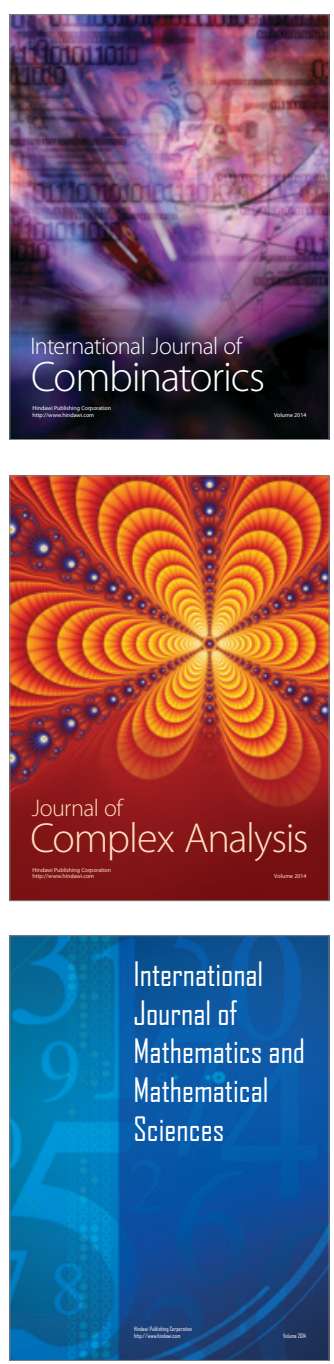
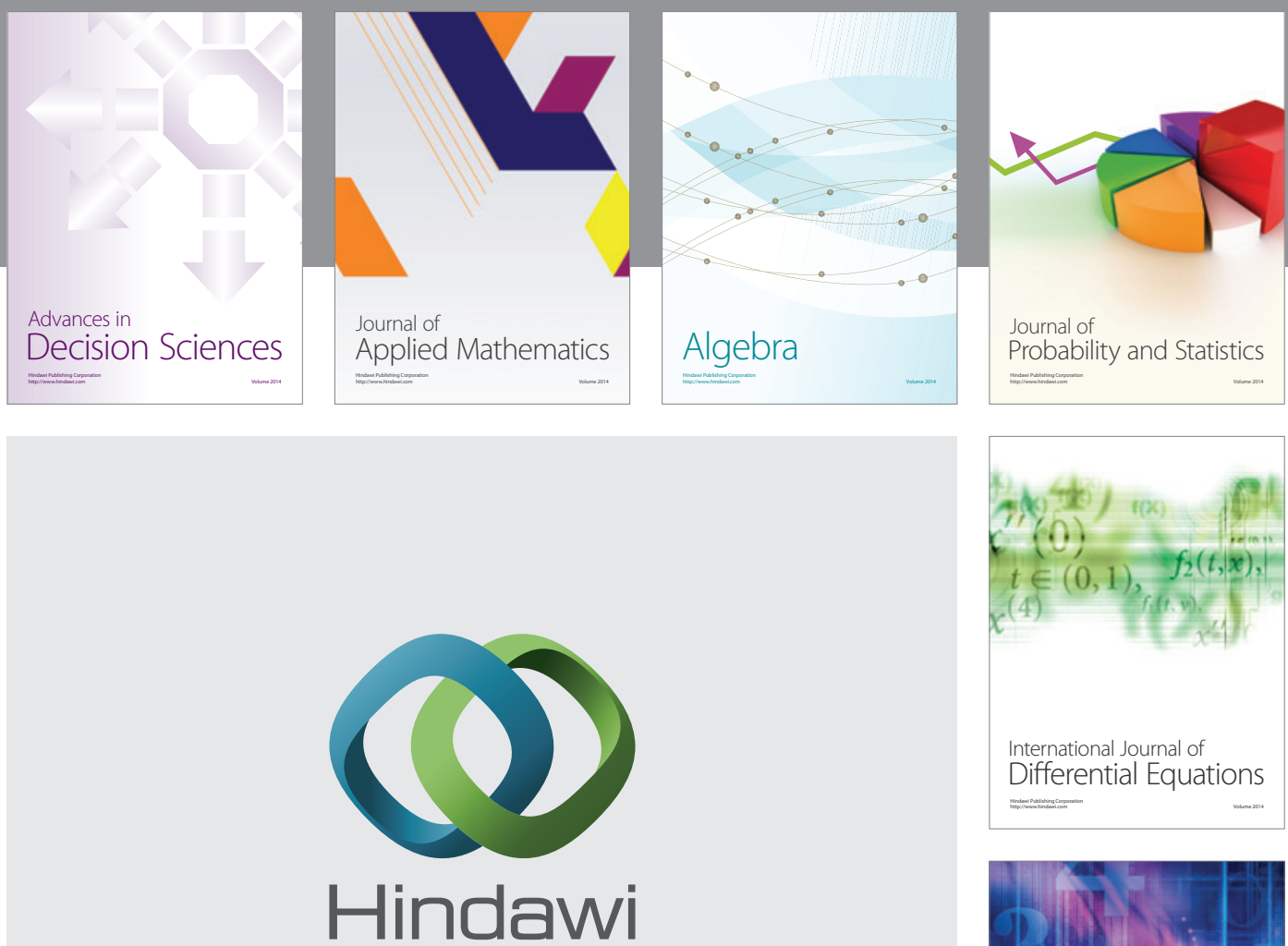

Submit your manuscripts at http://www.hindawi.com
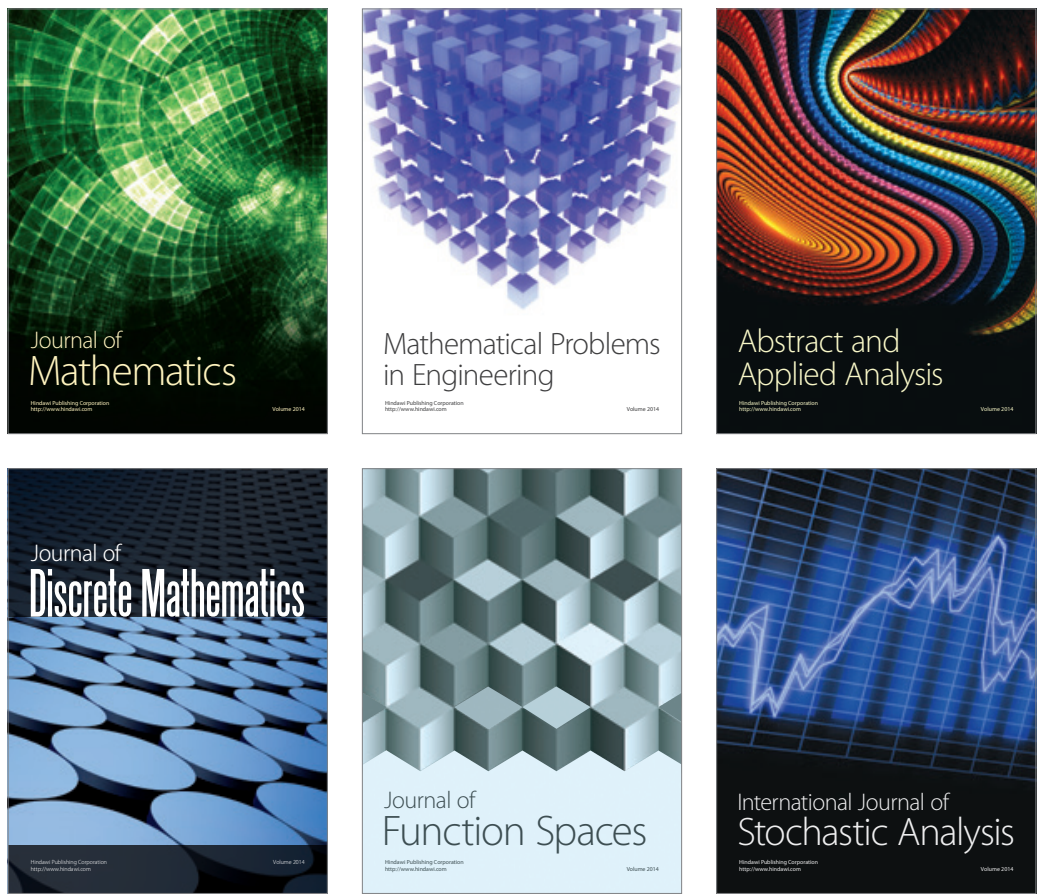

Journal of

Function Spaces

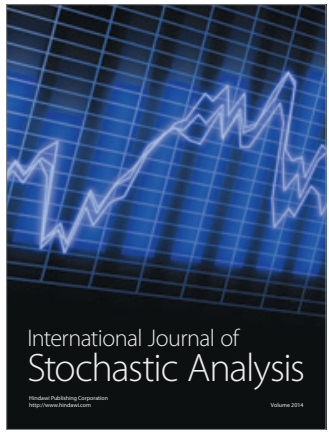

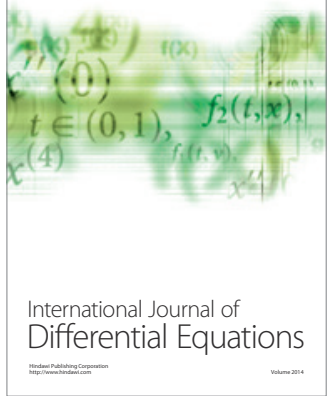
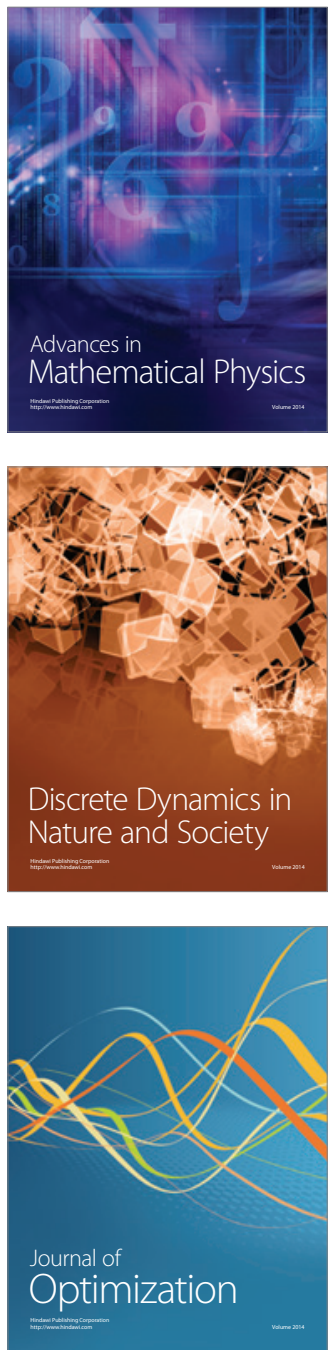